EPJ Web of Conferences 31, 00034 (2012)

DOI: $10.1051 /$ epjconf/20123100034

(C) Owned by the authors, published by EDP Sciences - SIF, 2012

\title{
Direct evidence of $\alpha$-particle condensation for the Hoyle state
}

\author{
Ad. R. Raduta ${ }^{1,2}$, B. Borderie ${ }^{1}$, E. Geraci ${ }^{3}$, N. Le Neindre ${ }^{1,4}$, \\ P. NAPOlitani ${ }^{1}$ and M. F. Rivet ${ }^{1}$ \\ for the ISOSPIN COLLABORATION
}
${ }^{1}$ Institut de Physique Nucléaire, CNRS/IN2P3, Université Paris-Sud 11, Orsay, France
${ }^{2}$ National Institute for Physics and Nuclear Engineering, POB-MG6, 077125 Bucharest-Măgurele, Romania
${ }^{3}$ INFN-Catania and Dipartimento di Fisica e Astronomia, Università di Catania, Italy
${ }^{4}$ LPC, CNRS/IN2P3, ENSICAEN, Université de Caen, Caen, France

\begin{abstract}
The fragmentation of quasi-projectiles from the nuclear reaction ${ }^{40} \mathrm{Ca}+{ }^{12} \mathrm{C}$ at $25 \mathrm{MeV} /$ nucleon was used to produce excited states candidates to $\alpha$-particle condensation. Complete kinematic characterization of individual decay events, made possible by the CHIMERA highgranularity $4 \pi$ charged particle multi-detector, reveals that $7.5 \pm 4.0 \%$ of the Hoyle state particle decays correspond to direct decays in three equal-energy $\alpha$-particles. Moreover, events with increased kinetic energy dispersion in the ${ }^{12} \mathrm{C}$ center of mass, which amount to $9.5 \pm 4.0 \%$, point toward the occurrence of a second competing molecular configuration, of a linear $\alpha$-chain.
\end{abstract}

Bose-Einstein condensation is known to occur in weakly and strongly interacting systems such as dilute atomic gases and liquid ${ }^{4} \mathrm{He}$. During the last decade it was theoretically shown that dilute symmetric nuclear matter may also experience particle condensation [1-3]. This new possible phase of nuclear matter may have its counterpart in low-density states of

This is an Open Access article distributed under the terms of the Creative Commons Attribution License 2.0, which permits unrestricted use, distribution, and reproduction in any medium, provided the original work is properly cited. 
self conjugate lighter nuclei, in the same way as superfluid nuclei are the finite-size counterpart of superfluid nuclear and neutron matter.

Convincing pleading in favour of $\alpha$-particle condensation in nuclei is provided by the excellent theoretical description of the Hoyle state (i.e. the first $0^{+}$excited state at $7.654 \mathrm{MeV}$ of ${ }^{12} \mathrm{C}$ ) and of the $0_{6}^{+}$state at 15.097 $\mathrm{MeV}$ of ${ }^{16} \mathrm{O}$ in terms of condensate type wave functions [4,5]. A note of caution has to be added though: according to recent Fermionic Molecular Dynamics (FMD) calculations [6] and pioneering works of Uegaki et al. [7], the Hoyle state has rather to be regarded as a mixture of configurations where exotic linear chains may exist among others.

From the experimental side, the detection of an $\alpha$-particle condensate expected to occur in the vicinity of the $N \alpha$-decay threshold [8] is a challenging task because of the few hundred-keV kinetic energies of emitted particles in the emitter center of mass which make the detection difficult. If, in order to overcome this drawback, one considers fast moving emitters as those obtained in projectile fragmentation, it is clear that high-granularity large-solid-angle-coverage detectors are necessary.

In this conference we report on recent results obtained out of the ${ }^{40} \mathrm{Ca}+{ }^{12} \mathrm{C}$ at $25 \mathrm{MeV} /$ nucleon nuclear reaction performed in 2003 at LNSCatania using the CHIMERA multi-detector [9]. The beam impinging on a thin carbon target $\left(320 \mu \mathrm{g} / \mathrm{cm}^{2}\right)$ was delivered by the Superconducting Cyclotron and its intensity was kept around $10^{7}$ ions/s to avoid pile-up events. While standard procedures $(E-\Delta E$ and shape identification) have been used for most reaction product identifications and energy calibration, dedicated energy calibrations based on TOF information were realized for $Z=1,2$ nuclei in order to achieve the best-possible precision. The average energy resolution is of about $2 \% . \alpha-\alpha$ and $\alpha-d$ correlations used to check the spectroscopic performances of our multi-detector revealed a satisfactory accuracy of few tens of $\mathrm{keV}$ in determining the centroids of the peaks corresponding to the ground and/or first exited states of ${ }^{8} \mathrm{Be}$ and, respectively, ${ }^{7} \mathrm{Li}[10]$. While this result may be certainly considered encouraging, we note that both correlated spectra and correlated functions manifest, because of finite angular and energy resolution, a peak broadening which gets more severe when the excitation energy increases.

Complete kinematical characterization of the reaction products allows one to identify the particle emitting sources using invariant velocity plots in the $\gamma \beta_{\perp}$ vs. $1 / 2 \log \left[\left(E+p_{\|}\right) /\left(E-p_{\|}\right)\right]$plane and show the dominant binary character of the collisions with the formation of a quasi-projectile (QP) and a quasi-target (QT). Here $E, p_{\|}, p_{\perp}$ stand for the energy and 
momentum components along and, respectively, perpendicular to the beam axis, $\beta=v / c$ is the reduced velocity and $\gamma=1 / \sqrt{1-\beta^{2}}$. The $\mathrm{QP}$ source may be easily isolated according to a rapidity of reaction products larger than $y_{\text {proj }} / 2$, where $y_{\text {proj }}$ is the projectile rapidity. Hereafter, we shall focus exclusively on QP decay products with an $\alpha$ multiplicity $m_{\alpha}=3$.

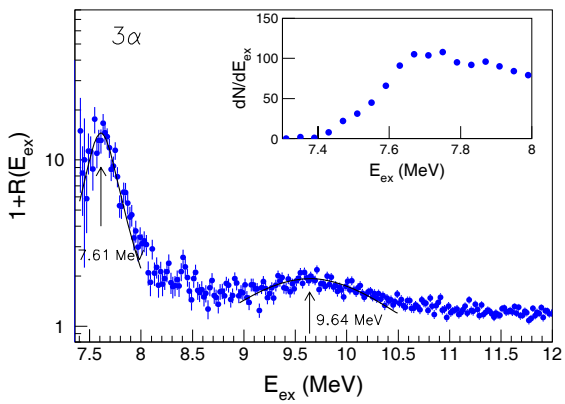

Figure 1: $3-\alpha$ correlation function as a function of excitation energy. The arrows correspond to centroids of Breit-Wigner distributions (solid lines). Inset: zoom of the correlated distribution in the energy domain of the Hoyle state.

Similar to two light-particle correlations, information on the $\alpha$ particle unstable excited states of ${ }^{12} \mathrm{C}$ populated by the ${ }^{40} \mathrm{Ca}+{ }^{12} \mathrm{C}$ at 25 $\mathrm{MeV} /$ nucleon reaction may be extracted from the $3 \alpha$-correlations. The $3 \alpha$ correlated spectrum and the correlation function $(\mathrm{CF})$ manifest two peaks. As one can see in fig. 1, in the case of $\mathrm{CF}$ the peaks are centered at $E_{e x}=7.61$ $\mathrm{MeV}(\Gamma=0.33 \mathrm{MeV})$ and $E_{e x}=9.64 \mathrm{MeV}(\Gamma=1.14 \mathrm{MeV})$ thus showing that by projectile fragmentation we have populated the Hoyle state $\left(E_{e x}^{e x p}=7.654\right.$ $\mathrm{MeV}, \Gamma^{e x p}=8.5 \mathrm{eV}$ ) and the complex excited region characterized by the strong $E_{e x}^{e x p}=9.64 \mathrm{MeV}\left(\Gamma^{e x p}=34 \mathrm{keV}\right), 3^{-}$state and by the broad $E_{e x}^{e x p}=10.3$ $\mathrm{MeV}, 0^{+}$state submerging a possible $2^{+}$state at $9.7 \mathrm{MeV}[11,12]$. We remind that the $\mathrm{CF}$ are defined as the ratio between the correlated (physical) yield $Y_{\text {corr }}$ and the product of single particle yields, generically termed as uncorrelated spectrum $Y_{\text {uncorr }}$, measured under the same conditions,

$$
1+R(X)=\frac{Y_{\text {corr }}(X)}{Y_{\text {uncorr }}(X)} .
$$

$Y_{\text {uncorr }}$ can also be built by mixing particles from different events as it is done in this work. The correlated spectrum allows one to estimate the ratio of background events (particles in coincidence originating from pysically uncorrelated events) with respect to genuine three-particle decays. For the exitation energy domain $7.37-7.97 \mathrm{MeV}$ and $m_{\alpha}=3$ this ratio is 1 .

The question which arises now is whether some of the $3 \alpha$-decays of the Hoyle state do correspond to an $\alpha$-particle condensate. Taking into account that according to the present understanding the existence of a condensate 

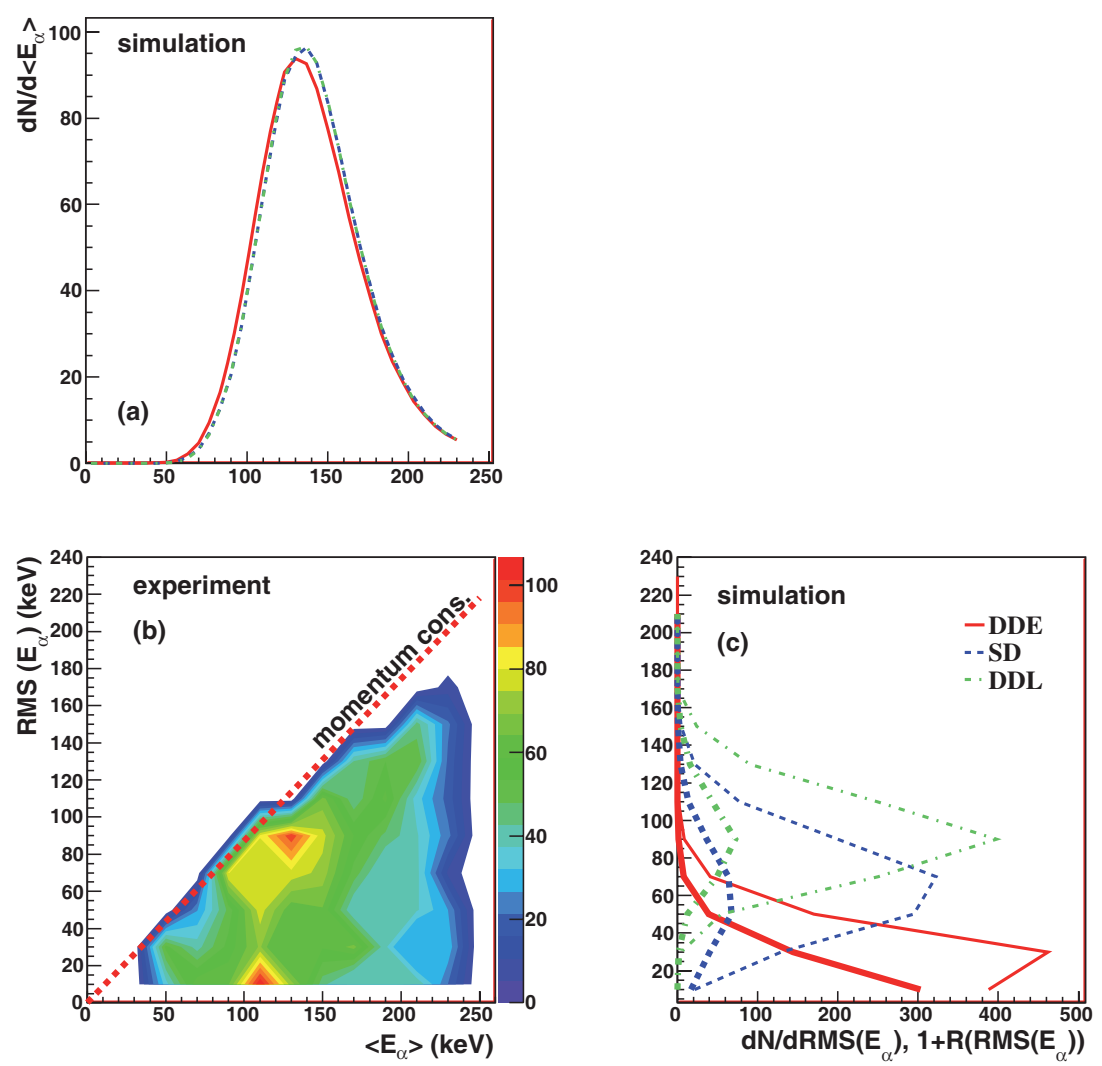

Figure 2: Three- $\alpha$ correlation function (b) expressed as a function of average kinetic energy - RMS of $\alpha$ particles corresponding to 1072 experimental events with $7.37 \leq E_{e x} \leq 7.97 \mathrm{MeV}$. The uncorrelated yield is built such as to allow for decay through ${ }^{8} \mathrm{Be}$. The dotted line marks the maximum RMS compatible with momentum conservation. $\left\langle E_{\alpha}\right\rangle$ (a) and RMS (c) spectra (normalized to 1072 events) of simulated DDE (solid lines), SD (dashed lines) and DDL (dot-dashed lines) decays of the Hoyle state after filtering through the detector replica with $R_{E}=2 \%$. Panel (c) presents also the RMS projection of $\left.\left.Y_{\text {corr }}\left(<E_{\alpha}\right\rangle, R M S\right) / Y_{\text {uncorr }}\left(<E_{\alpha}\right\rangle, R M S\right)$ (thick lines).

may be judged upon the occurence of simultaneous decays with equal kinetic energy particles, our task is to find such direct decay events and estimate their branching ratio. This may be done by comparing the experimental results with numerical simulations filtered by the multi-detector replica. Several decay channels have been considered: direct emission of three $\alpha$ particles with equal energies (DDE), sequential decays proceeding via the g.s. of ${ }^{8} \mathrm{Be}$ with isotropic emission of the two $\alpha$-particles from ${ }^{8} \mathrm{Be}$ (SD) 


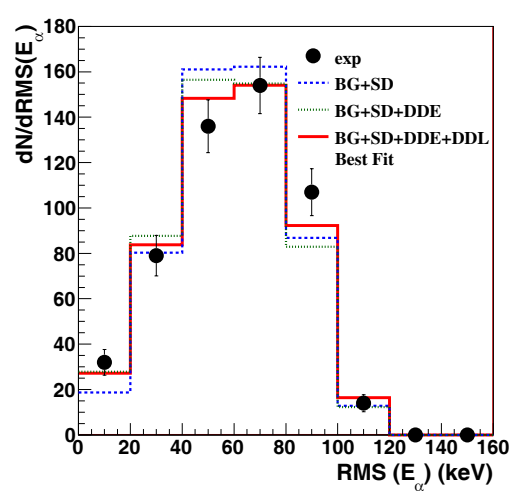

Figure 3: $R M S\left(E_{\alpha}\right)$ spectrum of simulated events corresponding to the best $\chi^{2}$ and $90 \leq<E_{\alpha}>\leq 150$ $\mathrm{keV}$ (solid line) in comparison with the corresponding experimental data (solid symbols). Dashed and dotted histograms illustrate simulation results if only sequential decay $(60 \%)$ and, respectively, sequential decay $(55.5 \%)+\operatorname{DDE}(4.5 \%)$ are considered. In all cases, background events amount to $40 \%$.

and direct decay from a linear chain (DDL). Limitation of direct decays (DD), which in principle assume random energy sharing among the emitted particles, to the particular sub-class of DDE is due to the predicted condensate nature of the Hoyle state. In each case and, for sequential decay, at each decay step the available energy $\left(E_{e x}+Q\right)$ is shared among the reaction products such as to conserve the linear and angular momenta and, eventually, obey to the postulated particularities of the decay. No preferential orientation between emitted particle velocities and source boost exists. The thus obtained reaction products are assumed to freely propagate toward the detector. Once the detector is reached, the events are filtered: if one or more particles hit a dead detector area or a detector which is out of work, the event is suppressed. If all particles have been correctly identified, the velocity vectors are altered such as to account for finite angular and energy resolution. After a first estimation of detection efficiencies ranging between $41 \%$ and $49 \%$, we have analyzed each decay pattern as a function of average kinetic energy and RMS in the ${ }^{12} \mathrm{C}$ c.m.. The spectra illustrated in the panels (a) and (c) of fig. 2 show that while the different decay mechanisms are practically indiscernable from the $\left\langle E_{\alpha}\right\rangle$ point of view, they may be discriminated using the RMS values. More precisely, for our average energy resolution of $2 \% Y_{\text {corr }}(R M S)$ of DDE, SD and DDL are peaked at 30, 70 and $90 \mathrm{keV}$. As a consequence, we adopt the best agreement between experimental and simulated $Y_{\text {corr }}\left(\left\langle E_{\alpha}\right\rangle, R M S\right)$ as the procedure to quantify each decay channel. For an optimum description of the experimental data, we add to pure simulated events the same proportion of background events that exists in the data. These are produced by partial event mixing (PEM) of experimental events; the technique of PEM which assumes that two particles belong to the same event while the third one comes from a different 
event is adopted in order to account for ${ }^{8} \mathrm{Be}$ decays. The comparison restricted to the excitation energy domain $7.4 \leq E_{e x} \leq 7.8 \mathrm{MeV}$ in order to reduce the importance of background events to $40 \%$ gets the best $\chi^{2}$ for 7.5 $\pm 4.0 \% \mathrm{DDE}$ and $9.5 \pm 4.0 \% \mathrm{DDL}$. This best $\chi^{2}$ value has been obtained after correcting for efficiencies; the error bars are estimated by taking into account statistical, $\chi^{2}$ and background errors. The $\operatorname{RMS}\left(E_{\alpha}\right)$ spectrum of simulated events (solid line) corresponding to the best $\chi^{2}$ and the energy domain $90 \leq<E_{\alpha}>\leq 150 \mathrm{keV}$ is represented in fig. 3 in comparison with the corresponding experimental data (solid symbols). Note that a limitation to DDE and SD (dotted histogram) as possible decays leads to a significant increase of $\chi_{\min }^{2}$ without changing the percentage of DDE. The existence of the direct decay channel was firstly investigated in Ref. [13] and its contribution to the total alpha decay (dominated by the ${ }^{8} \mathrm{Be}_{\text {g.s. }}+\alpha$ sequential decay) was estimated to be at most $4 \%$. The existence of such a decay channel is also of the greatest importance for nuclear astrophysics, due to the crucial role played by the Hoyle state for the synthesis of ${ }^{12} \mathrm{C}$ in the universe [14].

To visualize the results, fig. 2(b) presents the intra-event correlation function expressed as a function of $\left\langle E_{\alpha}\right\rangle$ and RMS corresponding to the selected 1072 events with $7.37 \leq E_{e x} \leq 7.97 \mathrm{MeV}$. Uncorrelated events are built by PEM but full event mixing (FEM) leads to a similar image. The relative separation of the peaks in terms of RMS allows us to identify the peak at low RMS $\leq 25 \mathrm{keV}$ as stemming from DDE, the broad peak at RMS $\approx$ $70 \mathrm{keV}$ as being due to SD and the high RMS $(\approx 90 \mathrm{keV})$ peak at $\left\langle E_{\alpha}\right\rangle$ $=120-140 \mathrm{keV}$ as coming from the direct decay of a linear chain with an $\alpha$ at rest and an equal sharing of the available energy between the other two $\alpha$-particles. We mention that the traditional way of identifying competing three-particle decays, that is the Dalitz plot, is not discriminative enough in the case of our data, because of high background contamination. Though the $\mathrm{CF}$ shows, as expected, a certain sensitivity to the event selection or event mixing recipe, the two observed peaks appear to be stable.

The same analyses has been performed for the much higher statistics region centered at $9.6 \mathrm{MeV}$ and no indication in favour of DDE has been obtained. In what regards the $0_{6}^{+}$state at $15.097 \mathrm{MeV}$ of ${ }^{16} \mathrm{O}$, the statistics is to poor to allow unambigous interpretation of low-RMS events.

In conclusion, the nuclear reaction ${ }^{40} \mathrm{Ca}+{ }^{12} \mathrm{C}$ at $25 \mathrm{MeV} /$ nucleon bombarding energy was used to produce states theoretically predicted as $\alpha$ particle condensate states. Supposing that equal values of kinetic energy of the emitted $\alpha$-particles represent a sufficient criterion for deciding in favour of $\alpha$-particle condensation, we found that $7.5 \pm 4.0 \%$ of events correspond- 
ing to the Hoyle state decay fulfil this criterion. To our knowledge, this is the first direct experimental indication of $\alpha$-particle condensation in nuclei. This study also evidenced the presence of direct alpha decays from a linear $\alpha$-chain. An experiment with higher statistics is planned to study the ${ }^{16} \mathrm{O}$ case.

\section{Acknowledgments}

The authors are indebted to P. Schuck for numerous discussions and one of the authors Ad. R. R. acknowledges the partial financial support from ANCS, Romania, under grant Idei nr. 267/2007.

\section{References}

[1] Röpke G. et al., Phys. Rev. Lett. 80 (1998) 3177.

[2] Beyer M. et al., Phys. Lett. B448 (2000) 247.

[3] Sogo T. et al., Phys. Rev. C 79 (2009) 051301.

[4] Tohsaki A. et al., Phys. Rev. Lett. 87 (2001) 192501.

[5] Funaki Y. et al., Phys. Rev. Lett. 101 (2008) 082502.

[6] Chernykh M. et al., Phys. Rev. Lett. 98 (2007) 032501.

[7] Uegaki E. et al., Prog. Theor. Phys. 57 (1977) 1262.

[8] Yamada T. and Schuck P., Phys. Rev. C 69 (2004) 024309.

[9] Pagano A. et al., Nucl. Phys. A734 (2004) 504.

[10] Raduta Ad.R. et al., Phys. Lett. B705 (2011) 65.

[11] Itoh M. et al., Nucl. Phys. A738 (2004) 268.

[12] Freer M. et al. Nucl. Phys. A834 (2010) 621c.

[13] Freer M. et al., Phys. Rev. C 49 (1994) R1751.

[14] Hoyle F. et al., Phys. Rev. 92 (1953) 1095; Cook C.W. et al., Phys. Rev. 107 (1957) 508. 